$\begin{array}{ll}\text { Research Square } & \text { Preprints are preliminary reports that have not undergone peer review. } \\ \text { They should not be considered conclusive, used to inform clinical practice, } \\ \text { or referenced by the media as validated information. }\end{array}$

\title{
Tolerability of MenACWY-TT Vaccination in Toddlers in the Netherlands; A Questionnaire Study
}

Jeanet Kemmeren ( $\sim$ jeanet.kemmeren@rivm.nl)

National Institute for Public Health and Environment https://orcid.org/0000-0002-4412-7615

L. van Balveren

Netherlands Pharmacovigilance Centre: Lareb

A. Kant

Netherlands Pharmacovigilance Centre: Lareb

$\mathrm{H}$ de Melker

National Institute for Public Health and the Environment: Rijksinstituut voor Volksgezondheid en Milieu

\section{Research article}

Keywords: MenACWY-TT vaccine, MenC-TT vaccine, toddlers, tolerability, reactogenicity

Posted Date: April 1st, 2021

DOI: https://doi.org/10.21203/rs.3.rs-366394/v1

License: (1) This work is licensed under a Creative Commons Attribution 4.0 International License. Read Full License 


\section{Abstract}

Background: In May 2018, the monovalent MenC-TT conjugate vaccine given at 14 months of age within the National Immunization Programme in the Netherlands was replaced by a 4-valent MenACWY-TT conjugate vaccine.

Methods: Results from a questionnaire about local reactions and systemic events within 4 days after vaccination were compared with data from a comparable tolerability study of a monovalent MenC-TT vaccine.

Results: The response rate was 5.5\% (1157/20966 questionnaires). Any local reaction was reported for $3.7 \%$ of the toddlers, with the highest percentage found for local redness at the injection site (2.9\%). Any systemic event was reported for $32.4 \%$ of the toddlers, with listlessness (22.4\%) most often reported. Fever was the only symptom more frequently reported after MenACWY-TT vaccination compared with MenC-TT vaccination (adj OR 1.61; 95\% Cl 1.29-2.01).

Conclusions: the 4-valent MenACWY-TT vaccine showed to be a little more reactogenic compared with to the monovalent MenC-TT vaccine, with a higher risk of fever within 4 days after vaccination. For the other factors no increased risks were found. Overall, this study shows that MenACWYTT vaccination is well tolerated in toddlers.

\section{Background}

Invasive meningococcal disease (IMD), caused by Neisseria meningitidis, is a life-threatening condition which, if left untreated, can lead to a case fatality ratio of up to $50 \%$ (1). After a steep rise of meningococcal C disease around 2000 in the Netherlands, all children between 1 and 19 years of age were vaccinated against meningococcal serogroup C disease (MenC) in a large campaign in 2002. From that point in time, MenC vaccination was included in the National Immunisation Programme (NIP) for children at 14 month of age, concomitantly with the first MMR vaccination. After 2002, a rapid decline of MenC IMD by more than $93 \%$ was observed. A decade after the introduction of the MenC vaccination, serogroup C IMD still remained at a very low level (2).

Since 2015, the Netherlands experienced an increase of serogroup W IMD (3). Before that time, MenW IMD was rare, with an average annual incidence 0.02 cases per 100,000. From 2015 the incidence started to increase reaching 0.5 IMD cases per 100,000 in 2017. In September 2017, the Dutch minister of Health decided to implement quadrivalent conjugate meningococcal (MenACWY-TT) vaccination to control the increase in serogroup W IMD. The target groups were toddlers aged 14 months where MenC conjugate vaccine (MenC-TT) was replaced by a MenACWY-TT conjugate vaccine and teenagers aged 13-14 years with a single MenACWY-TT vaccination (3).

The MenACWY-TT vaccine included in the NIP is licensed in the Netherlands in 2012 (4). Several clinical trials showed that this vaccine to be safe and well tolerated in toddlers, adolescents and young adults (5-17). However, after major changes in the NIP, the profile of frequently occurring adverse events (AEs) after vaccinations needs to be assessed as part of the policy in the Netherlands of monitoring safety and tolerability. Therefore, we evaluated the tolerability of the MenACWY-TT vaccination in toddlers in a questionnaire study about the occurrence of AEs, potential medical interventions and absence from childcare or work following vaccination. In addition, the results regarding the occurrence of AEs were compared with data from a previous tolerability study of MenC-TT vaccine which was conducted by the Netherlands Pharmacovigilance Centre Lareb several years ago.

\section{Methods}

\section{Study population and setting}

The study population consisted of two groups of healthy children, vaccinated with either monovalent MenC-TT or with quadrivalent MenACYW-TT around the age of 14 months, according to the NIP schedule in the Netherlands at the time of investigation. Data on local reactions and systemic AEs from the MenC-TT vaccinated group were obtained by the Netherlands Pharmacovigilance Center Lareb in 2014-2016 (report available on request). Data from the MenACWY-TT vaccinated group were collected from October 2018 to December 2019 and are described in the present study. The study design of both studies was similar.

Children from the southern part of the Netherlands were randomly selected from the vaccination register (Praeventis) of the RIVM (National Institute for Public Health and the Environment).

Parents of selected children received an invitation letter to participate in the study, together with a flyer with information on the study. Parents could register as participant at a website by creating an account until 4 days after the MenACWY-TT vaccination of their child. In the registration process, the scheduled vaccination date of the toddler was asked to be filled in and informed consent from their parents was obtained. 
Four days after the scheduled vaccination date, the parents received an email invitation to access the first questionnaire with their account on the website. This questionnaire asked about symptoms observed within four days after immunization (local reactions and systemic events). The invitation to fill in the second and third questionnaire was sent 14 and 28 days after the vaccination date, respectively. These questionnaires collected additional data on AEs not recovered after filling in the first questionnaire, and about systemic events related to the MMR vaccination. Results from these questionnaires will be reported separately.

A reminder was sent 2 days after the first questionnaire and 5 days after the second and third questionnaire.

\section{Vaccines}

The MenACWY-TT vaccine (Nimenrix ${ }^{\circledR}$ ) was given intramuscularly in the right arm. The 0.5 -ml dose contained Neisseria meningitidis-group $A$ polysaccharide $5 \mu \mathrm{g}$, Neisseria meningitidis-group C polysacharide $5 \mu \mathrm{g}$, Neisseria meningitidis-group W-135polysacharide $5 \mu \mathrm{g}$, and Neisseria meningitidis-group $Y$ polysacharide $5 \mu \mathrm{g}$, conjugated to tetanus toxoid carried protein $44 \mu \mathrm{g}$.

The MenC-TT vaccine (NeisVac-C®) was given intramuscularly in the right arm. The 0.5 -ml dose contained Neisseria meningitidis-group C polysacharide $10 \mu \mathrm{g}$, conjugated to tetanus toxoid carried protein $10-20 \mu \mathrm{g}$, absorbed on aluminium hydroxide, $0.5 \mathrm{mg} \mathrm{Al}^{3+}$ hydrated.

The 0.5 -ml dose of MMR vaccine contained $\geq 12,500$ p.f.u. mumps virus (Jeryl Lynn), $\geq 1,000$ p.f.u. measles virus, and $\geq 1,000$ p.f.u. rubella virus (M-M-R-Vaxpro®, MSD), and was administered subcutaneously in the left arm.

\section{Questionnaire}

The questionnaire included questions about the onset of local reactions and systemic events within four days after vaccination. Local reactions included swelling, redness, blue, induration, warmth, itch and pain at the injection site. Systemic events addressed sleeping problems, fever, being listless/apathetic, decreased appetite, vomiting, diarrhea, somnolence, rash, and other complaints. The presence was dichotomized (yes/no).

Information about the course of each symptom was also collected: time to onset, outcome, duration and impact (see Table 2 and Table 3 for grading scales). Additionally, the use of analgesics, occurrence of medical intervention, absence from childcare and/or other activities, and parents' or guardians' absence from work were asked.

Background information was collected regarding gender, medical history (i.e. positive when child regularly suffers from one or more of the following: coughing/shortness of breath, vomiting and/or diarrhea, infections, rash, hypersensitivity/allergy, other), number of children in household and attending daycare.

\section{Statistical analysis}

Assuming a percentage of fever of $8.2 \%$ (95\% Cl 6.7-10.1) in children participating in the MenC-TT study of Lareb, a $95 \%$ confidence level and a width of the confidence interval of $5 \%$, the sample size should be 1239 adolescents to be able to detect an absolute elevated risk of $1.5 \%$ (18). Assuming a response rate of $10 \%$ and a drop-out rate of $40 \%$, about 20600 parents were needed to be invited for participation.

Differences in baseline characteristics between the two study groups were calculated using the Chi-square test and Mann-Whitney $U$ test. The percentage of infants experiencing AEs within 4 days after immunization and $95 \%$ Cls were computed by type and severity of the AE. Binary logistic regression analysis was used to calculate odds ratios (ORs) for the relationship between local reactions and systemic AEs (dependent variables) and type of vaccine (independent variable) adjusted for gender, medical history, number of children in household, attending daycare Proportions of absence from child-care and/or other activities, parents' absence from work, and medical intervention within 4 days after vaccination were calculated with their $95 \%$ Cls.

Analyses were performed using SPPS statistics 24.

\section{Results}

\section{Response rate and population characteristics}

In total, 20966 invitations letters were sent to parents from children who were eligible for the MenACWY-TT vaccination. Of these, 1500 agreed to participate $(7,2 \%)$. The response rate for the questionnaire on symptoms within 4 days after vaccination was $5.5 \%$ ( $n=$ 1157) and a little lower than the response rate in the comparative MenC-TT study of Lareb $(6.4 \%(n=2727)$.

Of all respondents, $50.1 \%$ concerned male toddlers (see Table 1). In the MenACWY-TT study $76.9 \%$ of the children attended daycare, which is more than in the previous MenC-TT study (60.3\%; p 0.01$)$. In the MenACWY-TT study, a medical history was reported for $39 \%$ of the toddlers, with coughing/shortness of breath (17.9\%) and rash (16.1\%) as most reported. In the MenC-TT study, these comorbidities were less frequent (29.2\%), 
also with rash $(11,4 \%)$ and coughing/shortness of breath $(11.0 \%)$ most frequently reported. Furthermore, the distribution in season of vaccination was significantly different between the two groups $(\mathrm{p}<0.01)$.

Table 1

Characteristics of the participants

\begin{tabular}{|c|c|c|c|}
\hline Characteristics & Study population MenACWY-TT $(n=1157)$ & $\begin{array}{l}\text { Study population } \\
\text { MenC-TT }(n=2727)\end{array}$ & p-value \\
\hline Gender (n; \%) & $580(50.1)$ & $1401(51.4)$ & $P=0.48$ \\
\hline Male & $577(49.9)$ & $1326(48.6)$ & \\
\hline \multicolumn{4}{|l|}{ Female } \\
\hline Season of vaccination (n; \%) & $240(20.7)$ & $750(27.5)$ & $P<0.01$ \\
\hline Q1 & $329(28.4)$ & $436(16.0)$ & \\
\hline Q2 & $265(22.9)$ & $747(27.4)$ & \\
\hline Q3 & $323(27.9)$ & $794(29.1)$ & \\
\hline \multicolumn{4}{|l|}{ Q4 } \\
\hline Length in cm (mean; sd) & $77.9(3.2)$ & $78.4(3.3)$ & $P<0.01$ \\
\hline Weight in kg (mean; sd) & $10.1(1.1)$ & $10.2(1.3)$ & $P<0.01$ \\
\hline Number of children in household (n; \%) & $634(54.8)$ & $1518(55.7)$ & $P=0.35$ \\
\hline 1 & $403(34.8)$ & $932(34.2)$ & \\
\hline 2 & $99(8.6)$ & $197(7.2)$ & \\
\hline 3 & $12(1.0)$ & $40(1.5)$ & \\
\hline 4 & $1(0.1)$ & $13(0.5)$ & \\
\hline$>=5$ & $8(0.7)$ & $26(1.0)$ & \\
\hline \multicolumn{4}{|l|}{ Unknown } \\
\hline Attending daycare $(\mathrm{n} ; \%)$ & $264(22.8)$ & 1057 (38.8) & $P<0.01$ \\
\hline No & $890(76.9)$ & $1644(60.3)$ & \\
\hline Yes & $3(0.3)$ & $26(1.0)$ & \\
\hline \multicolumn{4}{|l|}{ Unknown } \\
\hline Comorbidity (n; \%) & $703(60.8)$ & $1930(70.8)$ & $P<0.01$ \\
\hline No & $454(39.2)$ & $797(29.2)$ & \\
\hline Yes & & & \\
\hline
\end{tabular}

\section{Local reactions after MenACWY-TT vaccination}

Table 2 shows the frequency, severity, onset time and duration of local reactions that occurred within 4 days after vaccination. Within these period, parents reported one or more local reactions in 43 children (3.7\%) with a total of 103 local reactions reported. Injection site redness was the most commonly reported local reaction. Most of the reactions were recovering or had already recovered within 4 days after vaccination ( $88.3 \%$ ). The mean time of onset of the different local reactions was within 17 hours after vaccination and ranged from $0-72$ hours. The mean duration was 32.3 hours (range $0-72$ hours). None of the parents reported that local reactions had high impact. 
Reported Frequency of local reactions within 4 days after MenACWYTT vaccination

\begin{tabular}{|c|c|}
\hline Local reaction & $\begin{array}{l}\text { MenACWY-TT vaccination } \\
(n=1157)\end{array}$ \\
\hline Swelling (n; \%) & $21(1.8)$ \\
\hline Redness (n; \%) & $34(2.9)$ \\
\hline Pain (n; \%) & $8(0.7)$ \\
\hline Blue (n; \%) & $5(0.4)$ \\
\hline Induration (n; \%) & $18(1.6)$ \\
\hline Warmth (n; \%) & $15(1.3)$ \\
\hline Itch (n; \%) & 0 \\
\hline Other (n; \%) & $2(0.2)$ \\
\hline Size of the reaction $(\mathrm{cm})$ & $15(34.9)$ \\
\hline $1-2$ & $22(51.2)$ \\
\hline $3-5$ & $6(13.9)$ \\
\hline$>5$ & $29(67.4)$ \\
\hline Recovered from local reactions (n; \%) & $9(20.9)$ \\
\hline Yes & $4(9.3)$ \\
\hline No, but it is recovering & $1(2.3)$ \\
\hline No, not recovered & $32(74.4)$ \\
\hline Unknown & $11(25.6)$ \\
\hline Impact of the reaction $(n ; \%)$ & 0 \\
\hline No & $16.8(15.9 ; 0-72)$ \\
\hline Moderate & $32.3(20.3 ; 0-72)$ \\
\hline \multicolumn{2}{|l|}{ High } \\
\hline \multicolumn{2}{|l|}{ Mean onset time in $\mathrm{hr}$ (sd; range) } \\
\hline Mean duration in hr (sd; range) & \\
\hline
\end{tabular}

\section{Systemic AEs after MenACWY-TT vaccination}

Parents reported one or more systemic events for 32.4\% $(n=375)$ of the children. A total of 971 systemic AEs were reported. Of these symptoms, listlessness was most often reported (22.4\%), with a mean duration of 2.4 days. After that, sleeping problems (13.9\%) and fever (13.4\%) were most commonly reported (see Table 3). The mean onset time of systemic AEs ranged from $23.9-60.3$ hours and the mean duration ranged from 20.3-41.4 hours. From all children with a systemic AE, 61.8\% had recovered, or almost recovered within 4 days. The impact for almost all systemic AE ranged from none to moderate. Exceptions were sleeping problems and vomiting, with a high impact as reported by $9.9 \%$ and $8.1 \%$ of the parents, respectively. Children with a reported local reaction experienced more often systemic AEs (60.5\%) than children without a reported local reaction $(31.3 \%)(p<0.001)$. This association was particularly observed in case of sleeping problems $(p=0.04)$, listlessness $(p<0.001)$ and other AEs $(p=0.04)$.

Parents from children with a medical history reported significantly more often vomiting after vaccination than children without a medical history (OR 2.24, 95\% Cl 1.15-4.38). No other significant associations between adverse events and medical history were found. 
Reported frequency of systemic events within 5 days after MenACWY-TT vaccination $(n=1157)$

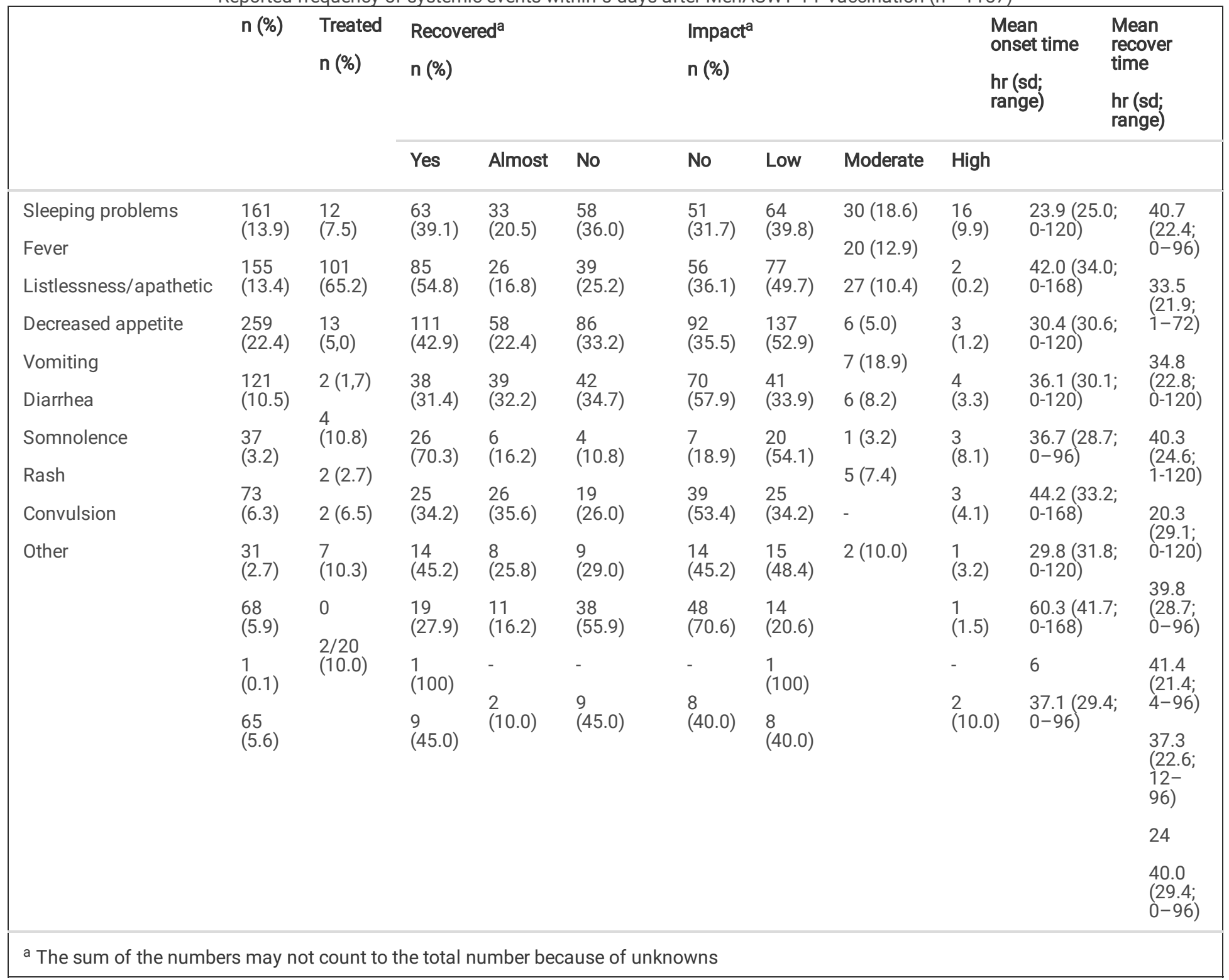

Absence and medical intervention after MenACWY-TT vaccination

Absence from attending childcare within 4 days after vaccination was reported in $10.2 \%$ of the children. The median duration was $1-2$ days (see Table 4).

From all parents or guardians, $5.5 \%$ were absent from work to take care of the vaccinated child, with a median of also $1-2$ days. 
Table 4

Frequencies in absence and medical intervention after MenACWY-TT vaccination

\begin{tabular}{|c|c|}
\hline & $\begin{array}{l}\text { After vaccination } \\
\mathrm{n}(\%)\end{array}$ \\
\hline Absence from childcare $(n=433)$ & $329(76.0)$ \\
\hline No & $16(3.7)$ \\
\hline Yes, $<1$ day & $22(5.1)$ \\
\hline Yes, $1-2$ days & $6(1.4)$ \\
\hline Yes, > 2 days & $60(13.9)$ \\
\hline Not applicable & $354(81.8)$ \\
\hline Absence from work $(n=433)$ & $8(1.8)$ \\
\hline No & $13(3.0)$ \\
\hline Yes, < 1 day & $3(0.7)$ \\
\hline Yes, $1-2$ days & $55(12.7)$ \\
\hline Yes, > 2 days & 948 (81.9) \\
\hline Not applicable & $209(18.1)$ \\
\hline Analgesic use & $40(19.1)$ \\
\hline No & $61(29.2)$ \\
\hline Yes & $47(22.5)$ \\
\hline Start analgesic use ( $n=209)$ & $61(29.2)$ \\
\hline $0-6$ hrs after vaccination & $95(45.5)$ \\
\hline 6-24 hrs after vaccination & 79 (37.8) \\
\hline $24-48 \mathrm{hrs}$ after vaccination & $35(16.7)$ \\
\hline$>48$ hrs after vaccination & $410(96.0)$ \\
\hline Duration of analgesic use $(n=209)$ & $7(1.6)$ \\
\hline$<1$ day & $7(1.6)$ \\
\hline $1-2$ days & $3(0.7)$ \\
\hline$>2$ days & \\
\hline Medical intervention ( $n=427)$ & \\
\hline No & \\
\hline Call to general practitioner/youth doctor & \\
\hline Visit general practitioner/youth doctor & \\
\hline Other & \\
\hline
\end{tabular}

Analgesics within 4 days after vaccination were used by $18.1 \%$ of the children. Almost all of them used paracetamol to treat fever and the median duration was less than 1 day (see Table 4). For eight children, the general practitioner or youth doctor was called for medical advice, and another twelve children visited the GP or youth doctor for their AEs. Main reasons for contacting the GP were fever ( $\mathrm{n}=7$; including one child with febrile convulsions, local reactions $(n=2)$, extensive crying $(n=2)$ and anxiousness of the parents $(n=2)$.

\section{Comparison AEs after MenACWY-TT vs. MenC-TT vaccination}

Fever was the only symptom with a higher risk after MenACWY-TT vaccination compared to MenC-TT vaccination (adj OR 1.61; 95\%Cl 1.29-2.01) (see Table 5). For local reactions as well as for the other systemic events, no increased risks were found after MenACWY-TT vaccination.

Table 5. Risk of AEFI within 4 days after MenACWY-TT vaccination compared to

MenC-TT vaccination

Page $7 / 11$ 


\begin{tabular}{|lll|}
\hline & OR unadj $(95 \% \mathrm{Cl})^{\mathrm{a}}$ & OR adj $(95 \% \mathrm{Cl})^{\mathrm{a}, \mathrm{b}}$ \\
\hline All Local Reactions & $1.12(0.77-1.62)$ & $1.08(0.74-1.57)$ \\
\hline Fever & $1.71(1.38-2.13)$ & $1.61(1.29-2.01)$ \\
\hline Decreased appetite & $0.92(0.74-1.15)$ & $0.88(0.70-1.11)$ \\
\hline Vomiting & $1.00(0.68-1.48)$ & $0.96(0.64-1.42)$ \\
\hline Diarrhea & $0.98(0.74-1.29)$ & $0.97(0.73-1.29)$ \\
\hline Listlessness/Somnolence & $0.91(0.77-1.07)$ & $0.88(0.74-1.04)$ \\
\hline Rash & $1.25(0.92-1.69)$ & $1.20(0.88-1.63)$ \\
\hline Convulsion & $0.59(0.06-5.27)$ & $0.76(0.08-6.94)$ \\
\hline All systemic events & $0.96(0.83-1.11)$ & $0.93(0.80-1.08)$ \\
\hline a Ref category MenC-TT vaccination & \\
\hline b Adjusted for gender, comorbidity, number of children in household, attending daycare \\
\hline
\end{tabular}

\section{Discussion}

In the present study we evaluated the tolerability of the newly introduced MenACWY-TT vaccine in the NIP in toddlers. Our results show that solicited systemic AEs like listlessness, fever and sleeping problems frequently occur, although most of them recovered within 4 days. The frequency of rash was much lower, but the majority of the children had not recovered after 4 days. However, rashes are also a known adverse event of MMR vaccination which usually occurs 5-21 days after vaccination. Therefore, overlapping frequencies of this adverse event from both vaccines may explain the longer duration. A follow-up questionnaire showed that only 2 children experiencing rash within 4 days after vaccination, did not recover within 30 days after vaccination (data not shown). The impact for most of the systemic AEs was mild to moderate, but parents reported on a high impact of sleeping problems and the vomiting, while hardly mentioning problems with local reactions.

The tolerability of MenACWY-TT observed in our study was in line with that observed in Lareb's MenC-TT study conducted a few years earlier in a similar group of young children in the Netherlands. Only for fever a higher risk after MenACWY-TT vaccination was found within 4 days after vaccination.

The frequencies of local reactions we found in our study are remarkable lower compared with the results from post-licensure trials. In our study, redness was the most reported local reaction (2.9\%), whereas in other studies percentages up to $45 \%$ were found for local reactions $(10,12,19-$ 21). In the clinical trials, irritability was one of the most mentioned solicited general symptoms with frequencies between 15 and $45 \%(10,12,19$, 20), whereas in our study, listlessness was the most reported systemic AE (22.4\%). Unfortunately, we did not include irritability in our questionnaire, whereas the trials did not describe listlessness. Drowsiness was another frequently mentioned systemic AE in the trials (15-35\%), whereas in our study somnolence was hardly reported $(2.7 \%)$. On the other hand, the results for loss of appetite (10.5\% in our study vs. $10-25 \%$ in the trials) and fever ( $13.4 \%$ vs. $5-30 \%$ ) were more consistent. Differences in study design (cross-section vs RCTs, data collection, age distribution) may contribute to the differences in our results and the results from clinical trials. This is underlined by the fact that our results are very similar to the results of Lareb's MenC-TT study, which had the same study design. Only for fever a significant difference was found between these studies (13.4\% for MenACWY vaccination and $8.2 \%$ for MenC-TT vaccination), although our result is in line with what is reported in the SMPC of this vaccine $(\geq 1: 10)(4)$.

There were a few number of limitations to this study. Compared to Lareb's MenC-TT study, significant differences were identified in baseline characteristics for comorbidity, attending daycare and seasonality. Although this may influence the frequency of for example fever, no different risk estimations were found between the adjusted and unadjusted analyses. Confounding by any of the covariates is therefore unlikely.

Data from the MenACWY-TT and Lareb's MenC-TT study were collected in different years. As the circulation of infectious agents differs between years, this may have influenced the frequency of some systemic events, like fever, diarrhea and vomiting. Since many of these events in our study recovered within 4 days, we assume that this effect is limited. However, the actual effect is unknown.

The non-response in both studies was high. A low response rate can give rise to sampling bias if the nonresponse is unequal among the participants regarding exposure and/or outcome. It is not clear to what extent this has occurred in this study. But since they have the same study design, it may be assumed that the reasons for non-response is similar for the MenACWY-TT and MenC-TT study. Therefore it is unlikely that the comparison of these studies is biased. 


\section{Conclusions}

This questionnaire-based study shows, in line with pre-licensure data, that MenACWY-TT vaccination has a good tolerability. Local reactions hardly occur. Most reported systemic AEs are listlessness, fever and sleeping problems. Most of the events were mild to moderate and transient but the impact of sleeping problems and vomiting was relatively high. The MenACWY-TT vaccine showed to be a little more reactogenic compared to the MenC-TT vaccine, expressed by a higher risk of fever within 4 days after vaccination. For the other factors no increased risks were found. Overall, our study shows that MenACWY-TT vaccination is well tolerated in toddlers.

\section{Abbreviations}

AEs Adverse events

IMD Invasive meningococcal disease

MenACWY-TT Meningococcal ACWY vaccine

MenC Meningococcal serogroup C disease

MenC-TT Meningococcal C vaccine

MenW Meningococcal serogroup W disease

NIP National Immunization Programme

RIVM National Institute for Public Health and the Environment

\section{Declarations}

\section{Ethics approval and consent to participate}

According to Dutch law (i.e., the Medical Research Involving Human Subjects Act (WMO)), internet-based surveys among healthy volunteers do not require formal medical ethical approval (www.ccmo.nl). However, all procedures performed in this study were in accordance with the ethical standard of the institutional and/or national research committee and with the 1964 Helsinki Declaration and its later amendments or comparable ethical standards.

Written informed consent was obtained from all adolescents included in the study and their parents.

\section{Consent for publication}

Not applicable

\section{Availability of data and materials}

The datasets used and/or analysed during the current study are available from the corresponding author on reasonable request.

\section{Competing interests}

The authors declare that they have no conflict of interest.

\section{Funding}

The present study was funded by the Ministry of Health, Welfare and Sport. They had no role in the design, collection, analysis, and interpretation of data. Neither did it have any role in the manuscript preparation at any stage and in the decision to submit it for publication.

\section{Authors' Contribution}

JK: Conception and design of the study, analyses, drafted the manuscript. LvB: Conception and design of the study, data collection, critical revision of the manuscript. AK: Conception and design of the study, critical revision of the manuscript. HdM: Critical revision of the manuscript and supervised the whole study process.

All authors have read and approved the manuscript.

\section{Acknowledgements}


The authors would like to thank Roger Venema (department DVP of the National Institute for Public Health and the Environment, RIVM) for providing the individual data from the national immunization register Præventis.

\section{References}

1. WHO. Meningococcal meningitis. https://www.who.int/news-room/fact-sheets/detail/meningococcal-meningitis 2018.

2. Bijlsma MW, Brouwer MC, Spanjaard L, van de Beek D, van der Ende A. A decade of herd protection after introduction of meningococcal serogroup C conjugate vaccination. Clin Infect Dis. 2014;59:1216-21.

3. Knol MJ, Ruijs WL, Antonise-Kamp L, de Melker HE, van der Ende A. Implementation of MenACWY vaccination because of ongoing increase in serogroup W invasive meningococcal disease, the Netherlands, 2018. Euro surveillance $:$ bulletin Europeen sur les maladies transmissibles = European communicable disease bulletin. 2018;23.

4. European Medicines Agency. https://www.ema.europa.eu/en/documents/product-information/nimenrix-epar-product-information_en.pdf.

5. Ostergaard L, Lebacq E, Poolman J, Maechler G, Boutriau D. Immunogenicity, reactogenicity and persistence of meningococcal A, C, W-135 and Y-tetanus toxoid candidate conjugate (MenACWY-TT) vaccine formulations in adolescents aged 15-25 years. Vaccine. 2009;27:161-8.

6. Knuf M, Kieninger-Baum D, Habermehl $P$, Muttonen $P$, Maurer $H$, Vink $P$, et al. A dose-range study assessing immunogenicity and safety of one dose of a new candidate meningococcal serogroups A, C, W-135, Y tetanus toxoid conjugate (MenACWY-TT) vaccine administered in the second year of life and in young children. Vaccine. 2010;28:744-53.

7. Baxter R, Baine Y, Ensor K, Bianco V, Friedland LR, Miller JM. Immunogenicity and safety of an investigational quadrivalent meningococcal ACWY tetanus toxoid conjugate vaccine in healthy adolescents and young adults 10 to 25 years of age. Pediatr Infect Dis J. 2011;30:e41-8.

8. Bermal N, Huang LM, Dubey AP, Jain H, Bavdekar A, Lin TY, et al. Safety and immunogenicity of a tetravalent meningococcal serogroups A, C, $W-135$ and $Y$ conjugate vaccine in adolescents and adults. Hum Vaccin. 2011;7:239-47.

9. Memish ZA, Dbaibo G, Montellano M, Verghese VP, Jain H, Dubey AP, et al. Immunogenicity of a single dose of tetravalent meningococcal serogroups $A, C, W-135$, and $Y$ conjugate vaccine administered to 2- to 10-year-olds is noninferior to a licensed-ACWY polysaccharide vaccine with an acceptable safety profile. Pediatr Infect Dis J. 2011;30:e56-62.

10. Vesikari T, Karvonen A, Bianco V, Van der Wielen M, Miller J. Tetravalent meningococcal serogroups A, C, W- 135 and $Y$ conjugate vaccine is well tolerated and immunogenic when co-administered with measles-mumps-rubella-varicella vaccine during the second year of life: An open, randomized controlled trial. Vaccine. 2011;29:4274-84.

11. Dbaibo G, Macalalad N, Reyes MR, Dimaano E, Bianco V, Baine Y, et al. The immunogenicity and safety of an investigational meningococcal serogroups A, C, W-135, Y tetanus toxoid conjugate vaccine (ACWY-TT) compared with a licensed meningococcal tetravalent polysaccharide vaccine: A randomized, controlled non-inferiority study. Hum Vaccin Immunother. 2012;8.

12. Vesikari T, Forsten A, Boutriau D, Bianco V, Van der Wielen M, Miller JM. Randomized trial to assess the immunogenicity, safety and antibody persistence up to three years after a single dose of a tetravalent meningococcal serogroups $A, C, W-135$ and $Y$ tetanus toxoid conjugate vaccine in toddlers. Hum Vaccin Immunother. 2012;8:1892-903.

13. Klein NP, Baine Y, Bianco V, Lestrate PR, Naz A, Blatter M, et al. One or two doses of quadrivalent meningococcal serogroups $A$, C, W-135 and $Y$ tetanus toxoid conjugate vaccine is immunogenic in 9- to 12-month-old children. Pediatr Infect Dis J. 2013;32:760-7.

14. Leonardi M, Latiolais T, Sarpong K, Simon M, Twiggs J, Lei P, et al. Quadrivalent meningococcal (MenACWY-TT) conjugate vaccine or a fourth dose of $\mathrm{H}$. influenzae-N. meningitidis $\mathrm{C} / \mathrm{Y}$ conjugate vaccine (HibMenCY-TT) is immunogenic in toddlers who previously received three doses of HibMenCY-TT in infancy. Vaccine. 2015;33:933-41.

15. Borja-Tabora CF, Montalban C, Memish ZA, Boutriau D, Kolhe D, Miller JM, et al. Long-term immunogenicity and safety after a single dose of the quadrivalent meningococcal serogroups $A, C, W$, and $Y$ tetanus toxoid conjugate vaccine in adolescents and adults: 5-year follow-up of an open, randomized trial. BMC Infect Dis. 2015;15:409.

16. Bona G, Castiglia P, Zoppi G, de Martino M, Tasciotti A, D'Agostino D, et al. Safety and immunogenicity of a CRM or TT conjugated meningococcal vaccine in healthy toddlers. Vaccine. 2016;34:3363-70.

17. Cutland CL, Nolan T, Halperin SA, Kurugol Z, Ahmed K, Perrett KP, et al. Immunogenicity and safety of one or two doses of the quadrivalent meningococcal vaccine MenACWY-TT given alone or with the 13-valent pneumococcal conjugate vaccine in toddlers: A phase III, open-label, randomised study. Vaccine. 2018;36:1908-16.

18. Connor RJ. Sample size for testing differences in proportions for the paired-sample design. Biometrics. 1987;43:207-11.

19. Knuf M, Romain O, Kindler K, Walther U, Tran PM, Pankow-Culot $\mathrm{H}$, et al. Immunogenicity and safety of the quadrivalent meningococcal serogroups A, C, W-135 and Y tetanus toxoid conjugate vaccine (MenACWY-TT) in 2-10-year-old children: results of an open, randomised, controlled study. Eur J Pediatr. 2013;172:601-12.

20. Dbaibo G, Tinoco Favila JC, Traskine M, Jastorff A, Van der Wielen M. Immunogenicity and safety of MenACWY-TT, a meningococcal conjugate vaccine, co-administered with routine childhood vaccine in healthy infants: A phase III, randomized study. Vaccine. 2018;36:4102-

Page $10 / 11$ 
11.

21. Vesikari T, Forsten A, Bianco V, Van der Wielen M, Miller JM. Immunogenicity, Safety and Antibody Persistence of a Booster Dose of Quadrivalent Meningococcal ACWY-tetanus Toxoid Conjugate Vaccine Compared with Monovalent Meningococcal Serogroup C Vaccine Administered Four Years After Primary Vaccination Using the Same Vaccines. Pediatr Infect Dis J. 2015;34:e298-307.

\section{Supplementary Files}

This is a list of supplementary files associated with this preprint. Click to download.

- Questionnaire.docx 